\title{
COVID-19 LOCKDOWN LANDSLIDES: THE NEGATIVE IMPACT OF SUBSEQUENT LOCKDOWNS ON LONELINESS, WELLBEING, AND MENTAL HEALTH OF AUSTRALIANS
}

\author{
Kim Louise Johnston ${ }^{1 *}$, Jessica Lee Oliva² \\ 1. School of Psychological Sciences, Monash University, Victoria, Australia \\ 2. College of Healthcare Sciences, James Cook University, Queensland, A ustralia
}

Correspondence:kim.johnston@monash.edu

\begin{abstract}
OBJECTIVE

We previously reported on loneliness, depression, anxiety and stress of Australians living alone during the first COVID-19related government enforced lockdown in Australia. At this time, those living alone were experiencing relatively low levels of emotional distress. Since then, one state, Victoria, underwent a second extended lockdown period and until now, it was unclear what impact this sequential lockdown might have had on the mental health and wellbeing of Victorian citizens. The current study aimed to add to the emerging literature on the lockdown experience in Australia by directly comparing the levels of anxiety, depression, stress, loneliness, and well being between Victorians in the second extended lockdown and Australians in the firstlockdown.
\end{abstract}

\section{DESIGN}

Data from our original study of 384 Australians was compared with cross-sectional surveys of 340 Victorians during the second lockdown period.

\section{SETTING}

An online survey was administered with people residing in Victoria self-selecting to complete the study.

\section{OUTCOME MEASURES}

Participants were asked to complete the Depression, Anxiety and Stress Scale (DASS-21), WHO-5 Wellbeing Scale, and the University of California Los Angeles (UCLA) Loneliness Scale. They were also invited to offer their insights in to how the second extended lockdown experience had differed from the first.

\section{RESULTS}

Independent samples t-tests revealed that Australians were significantly more depressed, anxious, stressed, and lonely, and experienced reduced psychological well being in the second lockdown compared to the first. However overall, the levels indicated mild psychological distress. Qualitative insights revealed impact on mental health and a feeling of increased restrictions during lockdown two.

\section{CONCLUSIONS}

Participants demonstrated adaptation to the lockdowns, providing support for the measures the Australian government have adopted to physically protect Australians from COVID-19. Management of the negative psychological impact through attention to wellbeing practices is however recommended in light of the increase in mental health concerns and likely further lockdown periods.

KEYWORDS: COVID-19, psychological wellbeing, loneliness, lockdown 
In addition to the physical and economic impact of the novel SARS-COV-2 Coronavirus pandemic, emerging evidence speaks of immense psychological impacts on communities. Government protective measures such as lockdown and physical distancing, while vital to stemming transmission of COVID-19, have been associated with rises in depression, anxiety, and other negative psychological consequences. The current study seeks to add to our understanding of the impact repeated lockdowns have had on psychological wellbeing and mental health in Australia.

In Australia, the sequalae of COVID-19 has been relatively minor in comparison to other countries, with approximately 1.6 million total cases and 3000 COVID deaths recorded at the end of 2021 [1]. This is likely due to the stringent measures taken by the Australian state governments to keep the Australian public physically safe from the virus. Widespread and extended lockdown procedures to reduce the spread of disease have been implemented by the Australian government twice; the first a Stage 3 lockdown across Australia beginning 23rd March and easing from 11 th May 2020, and the second in Victoria (Stage 4 in Metropolitan Melbourne and Stage 3 in regional areas) beginning August 2nd (metro) and August 5th (regional) and easing from September 14th, 2020. Evidence of the negative impact of lockdown measures on psychological wellbeing has been reported in early severely affected countries like China [2-3] and Italy [4], however a less clear picture emerges of the impact of lockdown in countries which have comparatively lower infection rates and deaths due to COVID-19. An April survey of 1491 adults in Australia conducted by Stanton and colleagues [5] indicated $26.5 \%$ of participants reported moderate to extremely severe levels of depressive symptoms, $13.5 \%$ moderate to extremely severe levels of anxiety, and $18.1 \%$ moderate to extremelysevere levels of stress, as measured by the Depression Anxiety and Stress Scale [DASS-21; 6]. Similar impact was reported in a survey of 5070 Australians conducted by Newby et al. [7] during the peak of the April lockdown, which found $55 \%$ of respondents felt their mental health had worsened a little, and $23 \%$ a lot. Other studies however have reported less impact; for example, a survey of 1599 Australians conducted by Rogers and Cruickshank (2020 unpublished pre-print) during the first lockdown in April was suggestive of 'somewhat' rather than 'a lot' of deterioration to mental

health, and many reported 'no change' (40-50\%) or even 'improvement' (6-17\%). Similarly, findings of our study during the first lockdown revealed that, on average, the mental health of those living alone were experiencing relatively low levels of emotional distress. [8]

In light of the mixed evidence, lack of studies on repeated lockdowns, and likely continuation of COVID-19 snap lockdowns, additional clarity as to the impact sequential lockdowns might have on the mental health and wellbeing of Australians would be of potential benefit for those tasked with management of staff. Therefore, the current study aimed to add to the emerging literature on the lockdown experience in Australia by directly comparing the levels of anxiety, depression, stress, loneliness, and wellbeing, between Victorians who experienced a second extended lockdown, and Australians in the first lockdown. It was hypothesised that in lockdown two comparative to lockdown one; (i) levels of anxiety, depression, and stress symptoms would be higher, (ii) loneliness would be higher, and (iii) overall psychological wellbeing would be lower. The research also aimed to identify how the second lockdown might be experientially different to the first, by adopting a mixed methodology design.

\section{METHOD}

\section{PARTICIPANTS}

A total of 384 participants from Australia completed the online survey administered during the first lockdown (54 men, 328 women, 1 non-binary gender, 1 unspecified, Mage $=51$ years, SDage $=15$ years, age range 23 to 89 years). Participants were required to be adults (18+), Australian residents / citizens, and live alone. Most participants $(81 \%)$ had a postgraduate or undergraduate university qualification. Data relating to where in Australia the participants resided was not collected.

The second study sampled 340 participants from the state of Victoria, Australia, who completed one of two online surveys administered during the second lockdown (30 men, 307 women, 3 non-binary gender, Mage $=47$ years, SDage $=15$ years, age range 20 to 87 years). To participate in one of the surveys the requirements were the same as those for the firstlockdown survey; to be adults (18+) Australian residents / citizens and live alone $(n=280)$. The other survey did not require participants to be living alone and was open to all Australian adults. Data relating to where in Australia the participants resided was collected and data 
from those in Victoria were included in this study. Most of these participants $(73 \%)$ had a postgraduate or undergraduate university qualification and resided in Metropolitan Melbourne (83\%) while the remainder resided in regional Victoria (17\%).

Only a small proportion of participants in both samples knew someone diagnosed with COVID-19 (n = 10 in lockdown one and $n=25$ in lockdown two), with one participant diagnosed themselves in lockdown two sample. Approximately one third of participants sampled during the second lockdown had received a test for COVID-19 that had come back negative. Data relating to negative tests was not collected at the time of the first lockdown.

\section{MEASURES}

Data for the current study was drawn from three separate surveys, each of which are part of larger projects, one of which has been published [8]. In all surveys, participants responded to a series of questions regarding their COVID19 experience. An opportunity was also provided for participants completing surveys in the second lockdown to respond to an open-ended question about how their experience of the second lockdown was different to their first.

The World Health Organization Well-Being Index [WHO-5; 9] is among the most widely used questionnaires assessing subjective psychological well-being. It is a short questionnaire consisting of 5 questions measured on a 6 point Likert scale, which tap into current subjective wellbeing of the respondents. These five questions assess positive mood (good spirits, relaxation), vitality (being active and waking up fresh and rested), and general interest (being interested in things) over the two weeks prior to completion. The WHO-5 has been applied successfully across a wide range of study fields and has been validated in a number of studies with regard to both clinical and psychometric validity [see review 10]. In the current study the scale demonstrated excellent overall reliability from all three surveys $(a=.88-.92)$.

The DASS-21 [6] is a 21 -item questionnaire rated on a 4-point Likert scale never, sometimes, often, and always. It was chosen as a widely accepted and psychometrically sound measure of mood which emphasises mood state over clinical diagnosis. As expected from previous research [e.g., 10] all subscales demonstrated excellent overall reliability $(a=.90-.92$ for Depression; $a=.80-.87$ for Anxiety; $a=.87-.91$ for Stress).

The 3-item University of California, Los Angeles (UCLA) Loneliness Scale [11] is a short version of the longer questionnaire developed by Russell et al. [12] Participants rated how often they felt they lacked companionship, were left out, or felt isolated during the respective lockdown periods, on a 4-point Likert scale never, rarely, sometimes, and often. Reliability coefficients were $a=.87$ and $a=.84$ in the first and second lockdown respectively.

As per the Office of National Statistics [13] recommendations, a direct, 1 -item measure of Ioneliness was also used whereby participants were asked to rate how often they had felt lonely during the lockdown, which was again rated on the same 4-point Likert scale as the UCLA Loneliness Scale. There was good convergent validity demonstrated between this directmeasure and the UCLA Loneliness scale at both timepoints, with correlations of $r=$ .82 and $r=.78$, respectively.

\section{DESIGN AND PROCEDURE}

A summary of our sampling and analytical procedures can be seen in Figure 1. The study utilised a cross-sectional nonexperimental survey design and was completed online by clicking on a secure link to a Qualtrics platform hosting the surveys. Ethics was approved for all three studies by the Monash University Human Research Ethics Committee. Promotion of the study occurred via social media and personal networks, with no payment or incentive offered for participation. Participants self-selected into the first lockdown survey between May 5th and 13th 2020 (COVID19 stage 3 lock-down period in all states of Australia). Individual states commenced their own easing of restrictions from May 11 th, 2020, however following a rise of COVID-19 cases in Metropolitan Melbourne and Mitchell Shire, these parts of Victoria re-entered stage 3 lockdown. On August 2nd a State of Disaster was declared and Metropolitan Melbourne entered a stage 4 lockdown. Three days later August 5th, regional Victoria (including Mitchell Shire) re-entered stage 3 lockdown. On September 14, a staged process for Victoria to come out of lockdown commenced. Participants self-selected into the second lockdown surveys between August 8th and 13th September 2020 (while Regional Victoria was under a COVID-19 stage 3 lock-down while Metropolitan Melbourne was under a COVID-19 stage 4 lockdown). During the lockdowns the only valid reasons to be out of one's house was i) shopping for food, ii) accessing medical 
services or providing care, iii) exercise, and iv) going to work as an "essential worker". Additional restrictions for stage four included a curfew whereby residents could not leave their house between the hours of 8pm-5am, only one hour of exercise was permitted per day, and residents could only move about within a $5 \mathrm{~km}$ radius, unless they had a permit. Mask wearing outside the house also became mandatory.

\section{DATA ANALYSIS}

Data was exported from Qualtrics to Statistical Package for Social Sciences (SPSS). Before the datasets were collated missing values analyses were conducted on each individual dataset and all missing datapoints were found to be missing completely at random. One missing datapoint for the anxiety subscale from the first lockdown, one missing datapoint for the stress subscale from the second lockdown, and two missing datapoints on the direct loneliness measure from the first lockdown were left as missing. A series of independent t-tests were conducted to compare wellbeing, depression, anxiety, and stress scores between the two lockdown periods.

\section{RESULTS}

\section{QUANTITATIVE ANALYSES}

Depression, anxiety, stress, and wellbeing data from both lockdown two surveys were combined and to ensure there were no differences between these two sets of data independent samples t-tests were run to compare means on all dependent variables. Histograms and Q-Q Plots indicated some minor deviation from normal distribution where scores were weighted towards a positive skew (i.e., higher scores) for the subscales of Depression, Anxiety and Stress in both samples, which is to be expected in non- clinical samples. Extreme outliers were checked using $z$ scores $\geq \pm 3.29$ and three found on the anxiety subscale, one from one dataset and two from the other. To ensure these data points was not exerting undue influence on the model, their Cook distances were checked and found to be $<1$, ranging between 0.02 and 0.14 . As they were deemed not to be exerting undue influence on the model, they were left unchanged. Levene's test of equality of variances indicated variances were similar for all subscale variables across the two datasets ( $p>05$ ). Independent samples t-tests revealed no differences between the two datasets $(p<.05$ ) on any of the subscales and so the data was pooled together for subsequent analyses. Loneliness data was only collected in one of the lockdown two studies and so no comparisons were required.

Descriptive analysis of the key variables is presented in Table 1. Scores on the DASS-21 were doubled to allow for normative comparison; mean scores indicated that in the week prior to survey, participants in lockdown two were experiencing greater distress than in lockdown one with depression scores progressing from mild to mild-moderate levels, anxiety progressing from normal to normal-mild levels, and stress increasing but remaining in the normal range. It is important to note that the DASS scores should be regarded as providing an individual's score on an underlying dimension therefore these cut-offs are provided purely for comparative purposes [1 1]. Scores on the WHO5 wellbeing scale were multiplied by 4 for interpretation as a percentage. At lockdown one participants reported an average level of wellbeing, however during lockdown two this had decreased to below average levels. Similarly, loneliness levels also increased from lockdown one to lockdown two, using both the 3 -item and the direct measure.

TABLE 1: MEANS, STANDARD DEVIATIONS, AND RANGES FOR STUDY VARIABLES, BY LOCKDOWN

\begin{tabular}{lllllllll}
\hline & \multicolumn{7}{l}{ Lockdown 1 } & \multicolumn{7}{l}{ Lockdown 2 } \\
& Range & M & SD & N & Range & M & SD & N \\
\hline Wellbeing & $4-100$ & 54.6 & 22.4 & 384 & $0-100$ & 44.8 & 22.9 & 340 \\
Depression & $0-42$ & 10.6 & 8.7 & 384 & $0-42$ & 13.7 & 10.2 & 340 \\
Anxiety & $0-36$ & 5.4 & 6.0 & 383 & $0-38$ & 7.4 & 7.6 & 340 \\
Stress & $0-42$ & 10.7 & 8.0 & 384 & $0-42$ & 13.6 & 9.3 & 339 \\
Loneliness (3) & $3-12$ & 8.2 & 2.6 & 384 & $3-12$ & 9.1 & 2.5 & 282 \\
Loneliness (1) & $1-4$ & 2.7 & 1.0 & 382 & $1-4$ & 3.0 & 1.0 & 282
\end{tabular}


To compare mean scores on wellbeing, depression, anxiety, stress, and loneliness between lockdown one and lockdown two a series of independent t-tests were run. Once again histograms and $Q-Q$ Plots indicated some minor deviation from normal distribution where scores are weighted towards higher scores for the wellbeing scale, however only for the lockdown one group. The distribution was more evenly spread at lockdown two. For the depression, anxiety and stress scales, scores were weighted towards lower scores in both lockdown groups. For Ioneliness, scores were weighted towards higher scores in lockdown two and a more even distribution in lockdown one. Extreme outliers were again checked using z scores $\geq$ \pm 3.29 and three high scorers were found in the first lockdown for depression, eight high scorers were found in the first lockdown and three in the second lockdown for anxiety, and three high scorers were found in the first lockdown for stress. To ensure these data points were not exerting undue influence on the models, their Cook distances were checked and found to be $<1$, ranging between 0.01 and 0.03 . As they were deemed not to be exerting undue influence on the models, they were left unchanged. Levene's test of equality of variances indicated variances were significantly different $(p<.05)$ for the depression, anxiety and stress subscales across the two lockdowns and so results are reported for variances not assumed.

Independent samples t-tests revealed significant differences between the two lockdowns on wellbeing, depression, anxiety, and stress, with wellbeing significantly decreasing and depression, anxiety, and stress significantly increasing (refer to Table 2).

TABLE 2: RESULTS OF COMPARISONS OF WELLBEING, DEPRESSION, ANXIETY, AND STRESS ACROSS THE TWO LOCKDOWNS

\begin{tabular}{|c|c|c|c|c|c|c|c|}
\hline Variables & $\begin{array}{l}\text { Mean } \\
\text { Difference }\end{array}$ & $\begin{array}{l}95 \% \mathrm{Cl} \\
\text { difference }\end{array}$ & of the & $\begin{array}{l}\text { SE } \\
\text { difference }\end{array}$ & $t$ & df & $\begin{array}{l}\text { Cohen } \\
d\end{array}$ \\
\hline Wellbeing & -9.79 & -13.10 & -6.48 & 1.69 & $5.81^{*}$ & 722.00 & 0.43 \\
\hline Depression & 3.10 & 1.71, & 4.49 & 0.71 & $4.38 \dagger^{*}$ & 669.37 & 0.33 \\
\hline Anxiety & 2.01 & 1.01 & 3.02 & 0.51 & $3.93 \dagger^{*}$ & 646.61 & 0.29 \\
\hline Stress & 2.90 & 1.62 & 4.18 & 0.65 & $4.46 十^{*}$ & 672.26 & 0.33 \\
\hline Loneliness ( 3 items) & 0.89 & 0.49 & 1.29 & 0.20 & $4.40^{*}$ & 664.00 & 0.34 \\
\hline Loneliness (1 item) & 0.32 & 0.17 & 0.47 & 0.08 & $4.13^{*}$ & 662.00 & 0.32 \\
\hline
\end{tabular}

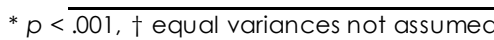

\section{QUALITATIVE ANALYSES}

To better capture the personal experiences of participants in the second lockdown the two authors of this paper independently identified themes on the first 10 cases, and through discussion created mutually agreed upon theme names and definitions that were used to independently code the next block of 10 cases. Then, their coding was compared, and theme names and definitions refined where necessary. This process continued until an acceptable inter-rater reliability $(r>$.80) was reached. Once achieved the first author completed the coding and then the second author reviewed it and any discrepancies were resolved through discussion. Blank responses ( $n=108$ ) and responses that did not adequately address the question ( $\mathrm{n}=17$ ) were not included in the sample size calculation for this analysis. Themes endorsed by $5 \%$ or more of participants are presented in Table 3. Percentages were rounded to the nearest whole number. 
TABLE 3: COMMON THEMES RELATING TO PEOPLES EXPERIENCES OF A SECOND LOCKDOWN

\begin{tabular}{|c|c|c|c|}
\hline Theme & Definition & Examples & $\begin{array}{l}\text { Frequency (\%) } \\
(\mathrm{N}=215)\end{array}$ \\
\hline $\begin{array}{l}\text { More restricted / } \\
\text { isolating }\end{array}$ & $\begin{array}{l}\text { Additional restrictions in } \\
\text { second lockdown have } \\
\text { resulted in feelings of being } \\
\text { more restricted and / or } \\
\text { isolated. }\end{array}$ & $\begin{array}{l}\text { It is worse than the first time, I } \\
\text { am isolating primarily at my } \\
\text { home where I live alone. I } \\
\text { am feeling very lonely and } \\
\text { imprisoned as I cannot see } \\
\text { any of my family. I also am } \\
\text { scared of travelling to see } \\
\text { my partner, so am only } \\
\text { seeing them once a week. }\end{array}$ & $85(40 \%)$ \\
\hline $\begin{array}{l}\text { Impact mental } \\
\text { health }\end{array}$ & $\begin{array}{l}\text { Exacerbation of existing } \\
\text { mental health concerns or } \\
\text { indicators of development of } \\
\text { anxiety / depression. }\end{array}$ & $\begin{array}{l}\text { Much harder, with a more } \\
\text { ambiguous endpoint. I have } \\
\text { suffered low mood and } \\
\text { anxiety this lockdown. }\end{array}$ & $50(23 \%)$ \\
\hline $\begin{array}{l}\text { No / minimal } \\
\text { difference }\end{array}$ & $\begin{array}{l}\text { Little or no difference } \\
\text { perceived comparative to } \\
\text { firstlockdown. }\end{array}$ & $\begin{array}{l}\text { No different l've virtually } \\
\text { stayed isolated when I'm not } \\
\text { working since the beginning. }\end{array}$ & 40 (19\%) \\
\hline Practical changes & $\begin{array}{l}\text { Changes related to everyday } \\
\text { life. }\end{array}$ & $\begin{array}{l}\text { More time working from } \\
\text { home. Less going out to do } \\
\text { basic shopping and when I } \\
\text { do go out it's only with } 2 \mathrm{~km} \\
\text { of where I live. }\end{array}$ & $20(9 \%)$ \\
\hline Iso fatigue & $\begin{array}{l}\text { Impact of ongoing COVID-19 } \\
\text { isolation. }\end{array}$ & $\begin{array}{l}\text { It's been harder in terms of } \\
\text { feeling like it's never ending } \\
\text { and not being sure when life } \\
\text { will ever get back to 'normal'. }\end{array}$ & $12(6 \%)$ \\
\hline Positives arisen & $\begin{array}{l}\text { Practical or emotional } \\
\text { benefits experienced. }\end{array}$ & $\begin{array}{l}\text { I am on disability pension } \\
\text { and have mental health } \\
\text { issues which make going out } \\
\text { stressful. Not having to make } \\
\text { excuses to stay in has been } \\
\text { restful. Also being able to } \\
\text { participate in events online - } \\
\text { church, gaming, therapy } \\
\text { sessions - generally gives me } \\
\text { all the human contact that I } \\
\text { want. }\end{array}$ & $10(5 \%)$ \\
\hline
\end{tabular}

Note. Frequencies were calculated by summing the number of participants who endorsed a theme. Individual participants were on ly able to endorse a theme once but could endorse more than one theme. 
This study aimed to articulate some of the indirect effects of the COVID-19 pandemic by exploring the impact of a second lockdown on the mental health and wellbeing of Australians. Findings indicated that although Australians reported normal levels of anxiety and stress and mild symptoms of depression during the first lockdown, those sampled during the second lockdown (which impacted only citizens in the state of Victoria) reported more concerns; with levels of depression, anxiety and stress all rising. These findings are consistent with an increase in loneliness and a decrease in wellbeing also reported between the two timepoints.

The qualitative analysis supported these findings, with almost a quarter of the sample (23\%) reporting negative impact on their mental health. Indicators of depressed mood such as "difficult to be motivated", "emotionally exhausting" and "much more depressing" were offered. Symptoms of anxiety were also described such as "more unsettled", "more worried about the future" and "bad insomnia and frequent wakings". One participant described what could be considered a complex trauma response: "I panicked, then felt fear for my life in the first lockdown. I felt over it, numb, couldn't care less, don't know how to feel, it is what it is, in the second lockdown." Some participants reported positive changes because of the second lockdown (5\%), however these tended to be more practical nature (e.g., working from home) rather than personal growth experienced in relation to the negative impact of the lockdown. Consistent with the relatively low levels of anxiety reported, post-traumatic growth is an unlikely outcome [see meta-analysis, 14], however this may be an important consideration for future research especiallyin more vulnerable populations.

In line with the increase loneliness scores, a sense of being more restricted and isolated was expressed by $40 \%$ of participants who responded to the open-ended questions. However, the theme of iso-fatigue was only minimally endorsed (6\%), suggesting that the sense of being more restricted and isolated may have arisen in part from the actual limitations imposed in the second lockdown (e.g., $5 \mathrm{~km}$ movement rule and curfew) rather than a perception that the second lockdown was more restrictive and isolating than the first.
It was highlighted that although the quantitative analysis indicated a decline in mood and wellbeing between lockdown one and two, $19 \%$ of the sample reported they felt no or minimal difference between the first and second lockdown. This finding is open to interpretation, but it may be due in part to a continuation of restrictive behaviour from the first lockdown as a self-imposed safety mechanism, therefore the second lockdown did not require substantial emotional or practical changes. As such, future research may like to explore the role of perceived control over external and internal states, and how this impacts subjective wellbeing. Overall, however, it would appear that there was an adaptation to the second lockdown given the normal-mild range of psychological distress and qualitative explanations.

These findings add to our emerging understanding of the impact of COVID-19 lockdowns on the mental health of Australians, which is of importance for management of staff in the public and health sectors as continuing lockdowns are likely to occur until widespread vaccination is in place. There are however a number of limitations associated with the current study which impact conclusions which can be drawn from the findings, such as the cross-sectional nature of the analyses and convenience sample which relied on people who had access to online social media forums. Further, the majority of the sample (82\%) were people who lived alone, so may not be reflective of all Victorian's experience. Moreover, while data from lockdown one included participants from all over Australia, data from lockdown two was restricted to participants from only one state, hence, it is possible that Victorian's have reduced indicators of mental health compared to the rest of the country, for reasons unrelated to COVID-19. This is however unlikely given that reports show Victorians to have comparable levels of depression, anxiety and stress as other states, with citizens of the state of Tasmania demonstrating the highest rates of depression and anxiety and citizens of the state of Queensland demonstrating the highest stress levels [15].

While some of the negative effects associated with repeated lockdowns are likely to dissipate as virus-related restrictions ease, increasing psychological distress may have more lasting impact, and further develop into mental health concerns [16]. The current study indicates a slight rise in concerns, suggesting that attention to staff wellbeing would be a valuable complement to current workplace 
safety systems especially in light of likely future lockdowns taking place. Workplace culture and recognition of COVID impact will be key in this regard, with managers tasked with creating a positive mental health environment which promotes ongoing self-care and destigmatises helpseeking. High-frequency pulse surveys which measure wellbeing could be used to share evidence on what works to improve psychological wellbeing as circumstances of the COVID-19 pandemic change. The findings also highlight the importance of exploring innovative ways to maintain connection and support during periods of lockdown and beyond. It is clear that attention to wellbeing as an integral component in Australia's recovery plan is needed for a sustainable and healthy future.

\section{References}

1. Australian Government Department of Health (2021). Coronavirus (COVID-19) case numbers and statistics. https://www.health.gov.au/health-alerts/covid19/case-numbers-and-statistics

2. Qiu, J., Shen, B., Zhao, M., Wang, Z., Xie, B., \& Xu, Y. (2020). A nationwide survey of psychological distress among Chinese people in the COVID-19 epidemic: Implications and policyrecommendations. General Psychiatry, 33(2). https://doi.org/10/1 136/gpsych-2020100213

3. Wang, C., Pan, R., Wan, X., Tan, Y., Xu, L., Ho, C. S., \& Ho, R. C. (2020). Immediate psychological responses and associated factors during the initial stage of the 2019 COVID-19 epidemic among the general population in China. International Journal of Environmental Research and Public Health, 17(5), 1729. https://doi.org/10.3390/iierph17051729

4. Rossi, R., Socci, V., Talevi, D., Mensi, S., Niolu, C., Pacitti, F., Marco, A. D., Rossi. A., Siracusano, A., \& Lorenzo, G. D. (2020). COVID-19 pandemic and lockdown measures: Impact on mental health among the general population in Italy. Frontiers in Psychiatry, 11. https://doi.org/10.3389/fpsyt.2020.00790

5. Stanton, R., To, Q. G., Khalesi, S., Williams, S. L., Alley, S. J., Thwaite, T. L., Fenning, A. S., \& Vandelanotte, C. (2020). Depression, anxiety and stress during COVID19: Associations with changes in physical activity, sleep, tobacco and alcohol use in Australian adults. International Journal Environmental Research in Public Health, 17, 4065.

https://doi.org/10.3390/ijerph17114065
6. Lovibond, S. H., \& Lovibond, P. F. (1995). Manual for the Depression Anxiety Stress Scales (2nd ed.).

Psychology Foundation.

https://doi.org/10.1037/†39835-000

7. Newby J. M., O'Moore, K., Tang, S., Christensen, H., \& Faasse, K. (2020) Acute mental health responses during the COVID-19 pandemic in Australia. PLOS ONE 15(7): e0236562.

https://doi.org/10.1371/journal.pone.0236562

8. Oliva, J. L., \& Johnston, K. L. (2021). Puppy love in the time of Corona: Dog ownership protects those living alone during the COVID-19 lockdown against Ioneliness. International Journal of Social Psychiatry, $67(3), 232-242$.

https://doi.org/10.1177/0020764020944195

9. Topp, C. W., Østergaard, S. D., Søndergaard, S., Bech, P. (2015). The WHO-5 Well-Being Index: A systematic review of the literature. Psychotherapy and Psychosomatics, 84(3):167-76. https://doi.org/10.1159/00037658 5

10. Crawford, J. R., \& Henry J. D. (2003). The Depression Anxiety Stress Scales (DASS): Normative data and latent structure in a large non-clinical sample. British Journal of Clinical Psychology, 42, 11-131. https://doi.org/10.1348/014466503321903544

11. Hughes, M. E., Waite, L. J., Hawkley, L. C., \& Cacioppo, J. T. (2004). A short scale for measuring loneliness in large surveys: Results from two population-based studies. Research on Aging, 26(6), 655-672. https://doi.org/10.1177/0164027504268574

12. Russell, D., Peplau, L. A., \& Cutrona, C. E. (1980). The revised UCLA Loneliness Scale: Concurrent and discriminant validity evidence. Journal of Personality and Social Psychology, 39, 472-480.

https://doi.org/10.1037/0022-3514.39.3.472

13. Office for National Statistics. (2018, December 5). Recommended national indicators of loneliness: Overview of our recommendations for national measures of loneliness.

www.ons.gov.uk/peoplepopulationandcommunity/w ellbeing/compendium/nationalmeasurementoflonelin ess/2018/recommendednationalindicatorsofloneliness

14. Shakespeare-Finch, J., \& Lurie-Beck, J. (2014). A metaanalytic clarification of the relationship between posttraumatic growth and symptoms of posttraumatic distress disorder. Journal of Anxiety Disorders, 28(2), 223-229. https://doi.org/10.1016/i.janxdis.2013.10.005 
15. Medibank (2016, September 7). How our mental health differs from state to state.

https://www.medibank.com.au/livebetter/health-

brief/health-insights/how-our-mental-health-differs-

from-state-to-

state/\#: : text=In\%20 contrast\%2C\%20the\%20lowest\%20

levels,levels\%2C\%20at\%20just\%2023.2\%25

16. Richter-Levin, G., \& XU, L. (2018). How could stress lead

to major depressive disorder? IBRO Reports, 4, 38-43.

https://doi.org/10.1016/i.ibror.2018.04.001 